\title{
Augmented Reality: A New Way to View the World
}

\author{
Manjul D E, Omkar Desai, Shrikar Desai \\ Department of Electronics Engineering \\ Shree L.R.Tiwari college of Engineering \\ Mumbai, India
}

\author{
Khushbu S. Tikhe \\ Asst. Professor, Department of Electronics Engineering \\ Shree L.R.Tiwari college of Engineering \\ Mumbai, India
}

\begin{abstract}
Technology in education can influence students to find out actively and may motivate them, resulting in an efficient process of learning. Although the physical world is threedimensional, mostly we opt to use two-dimensional media in education. The AR technology strengthens the effectiveness and attractiveness of teaching and learning for students in real scenarios. Since its introduction, augmented reality (AR) has been shown to own good potential in making the training process more active, effective and meaningful. This is possible because of its advanced technology that enables users to interact with virtual and real-time applications and brings them a natural experience. Augmented Reality could be a new medium, combining aspects from ubiquitous computing, tangible computing, and social computing. AR offers unique combining of physical and virtual worlds, with continuous and implicit control by the users for the purpose of viewing and interactivity. This study comprises of short introduction to augmented reality, AR components, working, and its comparison with virtual reality, advantages and its use in the field of education.
\end{abstract}

\section{Keywords: Augmented Reality, Augmented Reality in Education}

\section{INTRODUCTION}

Augmented Reality could be a style of video game that aims to duplicate the world's environment in an exceedingly computer. $\mathrm{AR}$ is an abbreviated name of Augmented Reality. An augmented reality system generates a composite view for the user that's the mix of the important scene viewed by the user and a virtual scene generated by the pc that augments the scene with additional information. The virtual scene generated by the $\mathrm{pc}$ is meant to reinforce the user's sensory perception of the virtual world they're seeing or interacting with. The goal of Augmented Reality is to make a system within which the user cannot tell the difference between the important world and also the virtual augmentation of it. Today Augmented Reality is employed in entertainment, preparation, engineering design, robotics, manufacturing and other industries.

Research on AR applications in education is still in an early stage, and there's a scarcity of research on the effects and implications of AR within the field of education. The use of AR has become more accessible because it no longer requires specialised equipment and should easily be used on mobile devices. The majority now own mobile devices, and therefore the use of those devices has increased, thereby enabling greater access to AR. The applications for mobile AR in education are increasing rapidly, and the feasibility of mobile AR has increased because of advances in mobile technology. AR mobile applications are available for several areas of education, and education related AR applications are now found on most of the mobile devices. Augmented reality (AR) may be a new technology that has emerged with potential for application in education. While a lot of research has been conducted on AR, few studies are conducted within the education field. The number of studies on AR is growing because of the effectiveness of this technology in recent years. AR has been utilized in different fields in education.

Teachers not only must spend a decent deal of private time working with computers but also should have a high level of innovation and confidence to use the new technologies that are embedded in contemporary education. The mixing of technology also provides a method to reinforce student learning and engagement in lectures. Therefore, recent studies have aimed to raised understand the applications adapted during lectures from the perspective of scholars, including multimedia, computer-based simulations, animations and statistical software (Neumann et al., 2011). Research by Geer and Sweeney (2012) showed that the utilization of a range of media applications to clarify concepts increased the understanding and supported greater collaboration between students. Many people now own mobile devices and thus have access to AR. the utilization of AR for learning has been made more feasible because of advances in mobile technology and therefore the increased use of smartphones. Smartphones and tablets are ideal to facilitate AR experiences, because of fast processors, graphics hardware, and various on board sensors.

Many people now own mobile devices and thus have access to AR. the utilization of AR for learning has been made more feasible because of advances in mobile technology and therefore the increased use of smartphones. Smartphones and tablets are ideal to facilitate AR experiences, because of fast processors, graphics hardware, and various on board sensors.

\section{AUGMENTED REALITY (AR) COMPONENTS}

\section{A. Scene Generator}

The scene generator is that the device or software to blame for rendering the scene. Rendering isn't currently one in all the key problems in AR, because some virtual objects have to be drawn, and that they often don't necessarily need to be realistically rendered so as to serve the needs of the applying.

\section{B. Tracking System}

The tracking system is one of the foremost important problems on AR systems mostly thanks to the registration problem. The objects within the real and virtual worlds must be properly aligned with relation to one another, or the illusion that the two worlds coexist are compromised. In industries, their applications demand accurate registration, especially for medical systems. 


\section{Display}

The technology for AR continues to be in development and solutions rely on design decisions. HMD (Head Mounted Display) is the most commonly used display devices for AR. Some other displays are Optical See through, Virtual Retinal Systems, Video See-Through, Monitor Based AR and Projector Based AR.

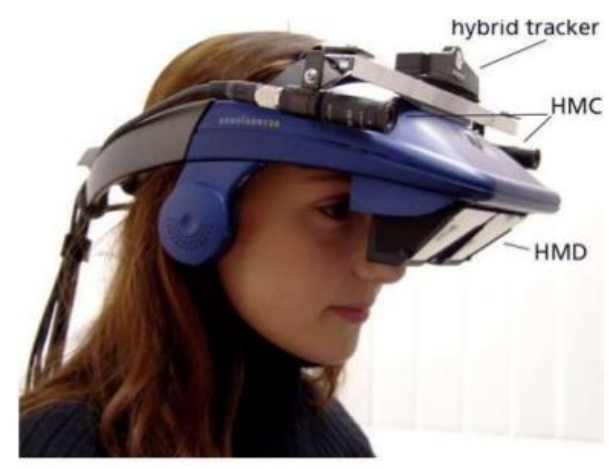

Fig. 1. Head Mounted - Display

When combining the reality and virtual world two basic choices are available: optical and video technology. Each of them has some trade-offs looking on factors like resolution, flexibility, field-of-view, registration strategies, among others. Display technology continues to be a limiting consider the event of AR systems.

See-through display glasses having sufficient brightness, resolution, field of view, and contrast to seamlessly blend a large range of real and virtual imagery are yet to be made. Furthermore, many technologies that begin to approach these goals aren't yet sufficiently small, lightweight, and low-cost. Nevertheless, the past few years have seen variety of advances in see-through display technology, as we shall see next.

\section{WORKING OF AUGMENTED REALITY}

The computer vision understands what's in the world around the user from the content of the camera feed. This enables it to point out digital content relevant to what the user is watching. The digital content is then displayed in an exceedingly realistic way, so it's a part of the important world - this can be called rendering.

Consider playing an augmented reality game employing a real box. First, computer vision processes the raw image from the camera, and recognizes the box. This triggers the sport. The rendering module augments the first frame with the AR game ensuring it precisely overlaps with the box. For this it uses the 3D position and orientation of the box determined by computer vision. Since augmented reality is live, all the above has got to happen anytime a replacement frame comes from the camera. Some phones work 30 frames per second, which provides us only 30 milliseconds to try and do all this. In many cases the AR feed we see through the camera is delayed by roughly $50 \mathrm{~ms}$ to permit all this to happen, but our brain does not notice it.

\section{DIFFERENCE BETWEEN AR AND VR}

Technology can change the way you take a look at the planet.

Augmented reality and computer game are two of the ways in which tech can change the way we take a look at the planet. The terms are confusing.

Sometimes people think AR and VR are the identical thing.

Augmented reality and computer game are increasingly utilized in technology, so knowing the difference is very important. Augmented reality is an enhanced version of reality made out of the utilization of technology to feature digital information of an object on a picture. AR is employed in apps for smartphones and tablets. AR apps uses our phone's camera to point out you a view of the real world ahead of us, then put a layer of data, including text and/or images, on top of that view. Apps can use AR for fun, like the sport Pokémon GO, or for information, like the app Layar. The Layar app shows an interesting information about places one can visit, using augmented reality. Open the app after visiting a site and browse information that appears in a very layer over our view. One can also find money machines, see property available, find restaurants, and more using the AR feature of the app. One can even discover new sites you probably did not know existed.

Virtual Reality is actually the use of technology to make a simulated environment. When we view VR, we are viewing a totally different reality than the one ahead of us. Virtual reality could also be artificial, like an animated scene, or an actual place that has been photographed and included in a very computer game app. With computer game, you'll be able to move around and appearance in every direction -- up, down, sideways and behind you, as if you were physically there. You can view computer game through a special VR viewer, like the Oculus Rift. Other computer game viewers use your phone and VR apps, like Google Cardboard or Daydream View. With computer game apps, you'll be able to explore places you've got never been, like the surface of Mars, the highest of Mt. Everest, or areas deep under the ocean. The big apple Times includes a computer game app that allows you to experience virtual environments on Earth and other planets. Google Earth also includes a computer game app.

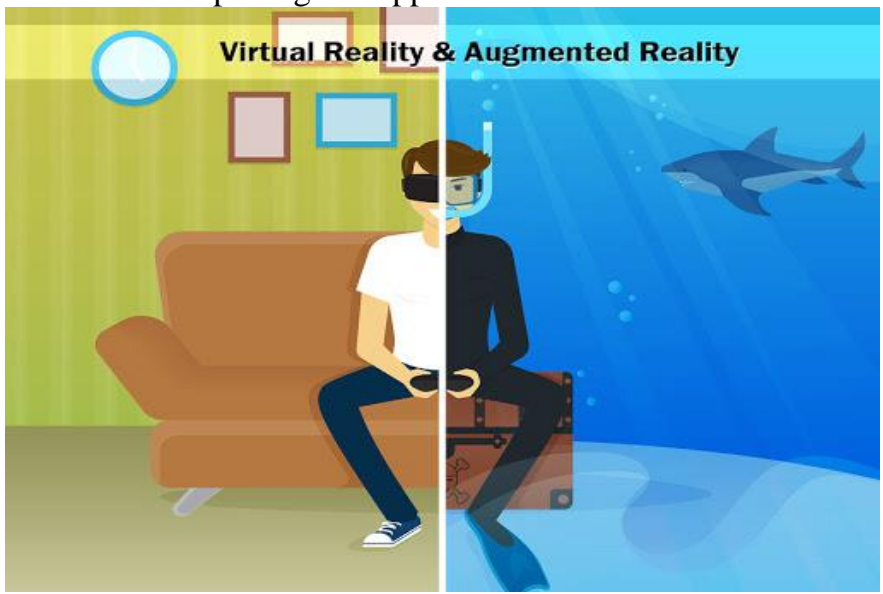

Fig. 2. Difference between VR and AR 


\section{AUGMENTED REALITY IN EDUCATION}

Augmented Reality technology isn't a brand new issue. it's been employed in fields such as: military, medicine, engineering design, robotic, tele-robotic, manufacturing, maintenance and repair applications, consumer design, psychological treatments, etc. (Azuma, Baillot, Behringer, \& Feiner, 2001). Displaying information by using virtual things that the user cannot directly detect along with his own senses can enable someone to interact with the real world in ways never before possible. We are able to change the position, shape, and/or other graphical features of virtual objects with interaction techniques augmented reality supports. Using our fingers or motions of handheld devices like shake and tilt we've got a capability to govern virtual objects, further on physical objects within the universe.

Augmented Reality are applied in the fields of learning, entertainment by enhancing a user's perception and interaction with the real world. User can move round the three-dimensional reflection and consider it from any viewpoint, a bit like a true object. The data conveyed by the virtual objects helps users perform universe tasks. Tangible Interface Metaphor is one in all the important thanks to improve learning. This property enables manipulation of three-dimensional virtual objects just by moving real cards without mouse or keyboard.

Augmented Reality also can be accustomed enhance collaborative tasks. It is possible to develop innovative computer interfaces that merge virtual and real worlds to boost face-to-face and remote collaboration.

These augmented reality applications are more the same as natural face-to-face collaboration than to screen based collaboration (Kiyokawa, et al., 2002).

Web technologies and internet are popular, as a practical situation people still prefer reading books rather than facing screens and textbooks are still widely used. Another application of this technology is in augmented reality textbooks. These books are printed normally but on focusing a webcam on the book brings out visualizations and interactive designs. This is often possible by installing special software on a computer, using special mobile apps or an online site.

The technology converts any existing book into an augmented reality edition after publication. Using 3D objects, miscellaneous and imaginative media, and simulations with differing kinds of interactions is the easiest ways of connecting two isolated worlds. Through the utilization of Augmented Reality in printed book pages, textbooks will became dynamic sources of knowledge, with this people with no computer background can still have a chic interactive experience.

The educational value of AR is closely linked to the way during which it's designed, implemented, and integrated into formal and informal learning environments. A crucial consideration is how AR technologies support and afford meaningful learning. Considering $A R$ as an idea instead of a particular form of technology would be productive for educators.

An educator's involvement is an important way to facilitate the development of favourable AR applications for teaching, which increases the potential of AR to be incorporated in education. AR applications are developed for many areas of education.

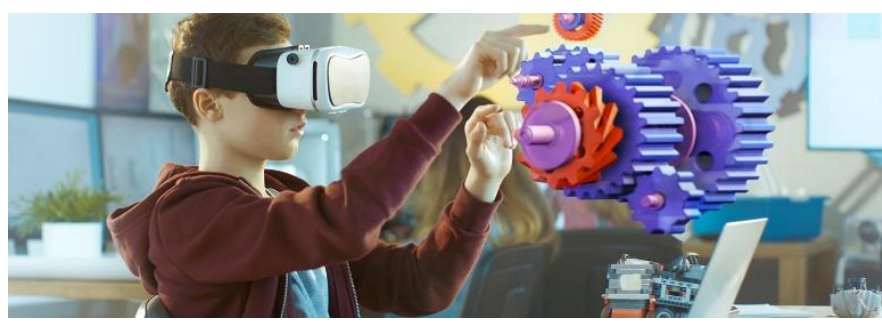

Fig. 3. In the field of education

\section{ADVANTAGES IN THE FIELD OF EDUCATION}

AR provides new ways of interacting with the real world and may create experiences that will not be possible in either a very real or virtual world. AR has the unique ability to form immersive hybrid learning environments that combine real and virtual objects. AR technologies enable users to experience scientific phenomena that does not seem to be possible within the globe, like certain chemical reactions, making inaccessible material available to students. The manipulation of virtual objects and observation of phenomena that are difficult to look at within the globe is facilitated through AR. this kind of learning experience can encourage thinking skills, and increase conceptual understanding of phenomena that are either invisible or difficult to look at similarly as correct any misconceptions. AR addresses learning difficulties that are often encountered with visualizing unobservable phenomena.

\section{CONCLUSION}

Augmented reality has power to alter how we use computers. Augmented reality makes the impossible possible and its potential in education is simply beginning. Augmented reality interfaces offer seamless interaction between the important and virtual worlds. Using augmented reality systems learners interact with the 3D information, objects and events during a natural way. The academic experience offered by Augmented Reality is different for variety of reasons as Mark Billinghurst (2002) mentioned:

AR supports interaction between real and virtual environments. It uses a tangible interface metaphor in object manipulation.

This review of the research conducted within the field of education shows that AR technology has the potential to be further developed in education. This can be because the benefits and beneficial uses of AR features are ready to engage students in learning processes and help improve their visualization skills. The features may also help teachers to clarify well and make the scholars easily understand what they teach. 
It is essential to coordinate with a team of specialist over possible augmented reality solution in educational issues. So as to realize realistic solutions we'd like to style and coordinate multi-disciplinary scientific research to reinforce content and environments. Educators can work with researchers to develop augmented reality interfaces. Software and hardware technologies play a vital and key role to provide augmented reality applications. Engineers can design different augmented reality environments. However for learning, in educational technology field, there's a giant need for instructional designers, who can design learning activities for augmented reality.

\section{REFERENCES}

[1] Nor Farhah Saidin, Department of Educational Sciences, Mathematics and Creative Multimedia, Faculty of Education, Universiti Teknologi Malaysia, 81310 Skudai, Johor, Malaysia (2015) "A Review of Research on Augmented Reality in Education: Advantages and Applications" http://dx.doi.org/10.5539/ies.v8n13p1

[2] Article in Procedia - Social and Behavioral Sciences - (December 2012) Mehmet Kesim, Yasin Ozarslan "Augmented Reality in Education: Current Technologies and the Potential for Education" CY-ICER2012

[3] "Introduction to Augmented Reality" R. Silva, J. C. Oliveira, G. A. Giraldi National Laboratory for Scientific Computation,

[4] Av. Getulio Vargas, 333 - Quitandinha - Petropolis-RJ Brazil

[5] "Augmented Reality: An Overview" Julie Carmigniani and Borko Furht

[6] J. Carmigniani

[7] Department of Computer and Electrical Engineering and Computer Sciences, Florida Atlantic University, Boca Raton, Florida, USA

[8] B. Furht (ed.), Handbook of Augmented Reality, DOI 10.1007/9781-4614-0064-6 1, (C) Springer Science+Business Media, LLC 2011

[9] "The Impact of an Augmented Reality Application on Learning Motivation of Students" (2019)

[10] Tasneem Khan, Kevin Johnston, and Jacques Ophoff

[11] Department of Information Systems, University of Cape Town, Cape Town 7701, South Africa

[12] Correspondence should be addressed to Jacques Ophoff; jacques.ophoff@uct.ac.za

[13] Copyright @ 2019 Tasneem Khan et al. 